Original Article

\title{
Prophylactic use of carvedilol to prevent ventricular dysfunction in patients with cancer treated with doxorubicin
}

\author{
Ahmed Mohamed Abuosa ${ }^{a}$, Ayman Hassan Elshiekh ${ }^{a}$, Kahekashan Qureshi ${ }^{b}$, \\ Mohammed Burhan Abrar ${ }^{\mathrm{b}}$, Mona A. Kholeif ${ }^{\mathrm{a}}$, Abdulhalim Jamal Kinsara ${ }^{\mathrm{a}, *}$, \\ Abdulwahab Andejani ${ }^{\mathrm{b}}$, Adel H. Ahmed ${ }^{\mathrm{a}}$, John G.F. Cleland ${ }^{\mathrm{c}}$ \\ ${ }^{a}$ King Saud bin Abdulaziz University for Health Sciences, COM-J, King Abdul Aziz Medical City-WR, King Faisal Cardiac Center, Saudi Arabia \\ ${ }^{\mathrm{b}}$ Princess Noorah Oncology Centre, Jeddah, Saudi Arabia \\ ${ }^{\mathrm{c}}$ National Heart \& Lung Institute, Royal Brompton \& Harefield Hospitals, Imperial College, London, United Kingdom
}

\section{A R T I C L E I N F O}

\section{Article history:}

Received 25 December 2017

Accepted 14 June 2018

Available online 18 June 2018

\section{Keywords:}

Doxorubicin

Carvedilol

Ejection fraction

Cardiomyopathy

\begin{abstract}
A B S T R A C T
Objective: Deterioration in ventricular function is often observed in patients treated with anthracyclines for cancer. There is a paucity of evidence on interventions that might provide cardio-protection. We investigated whether prophylactic use of carvedilol can prevent doxorubicin-induced cardiotoxicity and whether any observed effect is dose related.

Methods: A prospective, randomized, double-blind study in patients treated with doxorubicin, comparing placebo $(n=38)$ with different doses of carvedilol $[6.25 \mathrm{mg} / \mathrm{day}(\mathrm{n}=41), 12.5 \mathrm{mg} / \mathrm{day}(\mathrm{n}=38)$ or $25 \mathrm{mg} /$ day $(n=37)$ ]. The primary endpoint was the measured change in left ventricular ejection fraction (LVEF) from baseline to 6 months.

Results: LVEF decreased from $62 \pm 5 \%$ at baseline to $58 \pm 7 \%$ at 6 -months $(\mathrm{p}=0.002)$ in patients assigned to placebo but no statistically significant changes were observed in any of the 3 carvedilol groups. At 6 months, only one of 116 patients (1\%) assigned to carvedilol had an LVEF $<50 \%$ compared to four of the 38 assigned to placebo (11\%), ( $\mathrm{p}=0.013)$. No significant differences were noted between carvedilol and placebo in terms of the development of diastolic dysfunction, clinically overt heart failure or death. Conclusions: Carvedilol might prevent deterioration in LVEF in cancer patients treated with doxorubicin. This effect may not be dose related within the studied range.

(C) 2018 Published by Elsevier B.V. on behalf of Cardiological Society of India. This is an open access article under the CC BY-NC-ND license (http://creativecommons.org/licenses/by-nc-nd/4.0/).
\end{abstract}

\section{Introduction}

Anthracyclines are used to treat a wide spectrum of human malignancies but their use is associated with an increased risk of developing ventricular dysfunction that may be irreversible. Postchemotherapy left ventricular ejection fraction (LVEF) and cumulative anthracycline dose are independent correlates of its occurrence. Early detection of cardiotoxicity and its prompt treatment may be crucial for the preservation of cardiac function. ${ }^{1}$ Although the risk of cardiac dysfunction is proportional to the cumulative anthracycline exposure, $^{2}$ a substantial number of patients still develop severe cardiotoxicity at doses well below $550 \mathrm{mg} / \mathrm{m}^{2}{ }^{3}$ In a study on the early detection and prediction of cardiotoxicity, $27.6 \%$ of patients

\footnotetext{
* Corresponding author at: King Saud bin Abdulaziz University for Health Sciences, COM-J, King Abdul Aziz Medical City-WR, King Faisal Cardiac Center, Mail Code 6599, P.O. Box 9515, Jeddah 21423, Saudi Arabia.

E-mail address: kinsaraaj@ngha.med.sa (A.J. Kinsara).
}

developed chemotherapy-related cardiotoxicity. ${ }^{4}$ Another study found that doxorubicin in doses of less than $300 \mathrm{mg} / \mathrm{m}^{2}$ could induce cardiotoxicity. ${ }^{5}$ Strategies that might prevent chemotherapy-induced cardiomyopathy are receiving increased attention from oncologists and cardiologists. However, there is a dearth of randomized controlled trials reported to date. These include carvedilol which blocks $\beta 1, \beta 2$ and $\alpha 1$-adrenergic receptors with antioxidant properties, ${ }^{6}$ metoprolol, which only blocks $\beta 1$ receptors, perindopril (an angiotensin converting enzyme inhibitor $)^{7}$ and dexrazoxane, a chelating agent that is a derivative of ethylene diamine tetra acetic acid, which reduces the number of metal ions that can complex with anthracyclines and therefore the formation of superoxide free-radicals. ${ }^{8}$ Two randomized trials, have reported that once-daily, low-dose carvedilol reduces the development of ventricular dysfunction in patients treated for cancer with doxorubicin or epirubicin. ${ }^{9,10}$

Accordingly, we conducted a prospective, randomized, doubleblind, placebo-controlled dose-ranging study to assess the prophylactic use of carvedilol for preventing left ventricular dysfunction in cancer patients treated with doxorubicin. 


\section{Patients and methods}

This double-blind, randomized, placebo-controlled study was conducted at the King Faisal Cardiac Centre in King Abdulaziz Medical City-Jeddah, Saudi Arabia. The study was approved by a local institutional review board. Patients were randomly assigned to placebo or to one of three doses of carvedilol: $6.25 \mathrm{mg} \mathrm{OD}$, $12.5 \mathrm{mg}$ OD and $25 \mathrm{mg}$ OD. Randomization was performed by an independent statistician using computer generated random number numbers and allocation concealment was achieved with opaque sealed envelopes. Cancer patients aged $>16$ years who were treated with doxorubicin and met the inclusion and exclusion criterion were recruited for the study and were followed up for six months. We conducted the study to observe the early anthracycline cardiac toxicity (like all other similar trials), but late anthracycline toxicity needs many years of follow-up, which could be done, but with different protocol. Written informed consent was obtained from each participant.

Exclusion Criteria: Patients with left ventricular ejection fraction (LVEF) $<50 \%$ before enrolment to the study, known cardiomyopathy or on therapy for heart failure, bronchial asthma that required regular daily beta-2 stimulant therapy, severe peripheral arterial disease, second or third degree heart block, severe valvular heart disease, earlier therapy with anthracycline derivatives, coronary artery disease, thyroid function disorder and patients who were on beta-blockers or angiotensin convertingenzyme inhibitors were excluded.

Data were collected including a history, physical examinations, electrocardiograms and echocardiograms at baseline and after 2, 4 and 6 months after initiation of doxorubicin. Mainly twodimensional (2D) echocardiography was used and only in few cases, three-dimensional (3D) echo was done because 3D echo was not routinely available at our center. LVEF, and left ventricular diastolic (LVDd), and systolic diameters (LVSd) were measured from 2D echocardiograms by two consultant cardiologists, independently. An LVEF > 50\% was considered normal. Doppler ultrasound measures of LV diastolic dysfunction included the ratio of E (early mitral inflow velocity) to A (late mitral inflow velocity), mitral flow deceleration time (DT) and lateral E` on tissue Doppler imaging. Diastolic dysfunction was graded using the American Society of Echocardiography guidelines. ${ }^{11}$

\section{Statistical analysis}

The sample size calculation was based on the incidence of doxorubicin-induced subclinical cardiomyopathy at 6 months of $27.6 \%$ in the control and a $4 \%$ in the carvedilol group patients, reported in previous studies. ${ }^{4,9}$ The sample size was carried out based on alpha value, statistical power, and effect size. The effect size to detect reduction in the prevalence of doxorubicin-induced subclinical cardiomyopathy was estimated from previous studies to be $23.6 \%$. Using a 2 -sided $\alpha$ of 0.05 , a sample size of 37 patients per group in the study would provide $80 \%$ power to detect a $23.6 \%$ difference in the prevalence of cardiomyopathy between control and carvedilol groups. Statistical analyses were done by intentionto-treat. Continuous variables were reported as mean \pm standard deviations (SD) and categorical variables as frequencies and percentages. Chi-square or Fisher's exact tests were used to compare categorical variables. Baseline measurements of the four treatment groups were analyzed using one-way analyses of variance (ANOVA) and paired Student's $t$-test was used to compare baseline and last visit (month 6) values for each of the treatment groups with $\mathrm{p}<0.05$ considered statistically significant. The primary analyses of interest were the differences in the change in LVEF from baseline to six months for patients assigned to placebo compared to carvedilol, regardless of dose and then analysis for a carvedilol dose-response. Missing data were imputed using the last observation carried forward (LOCF) method.

The reproducibility of LVEF measurements was tested using intra-class correlation coefficients (ICC). A total of 20 subjects were randomly selected. Two independent observers were involved in reading the echocardiography images. To determine intra-observer reproducibility, the first observer performed two readings at two different time points. To determine inter-observer reproducibility, a second observer who was blinded to the results of the first observer, provided independent readings on the same subjects.

\section{Results}

\subsection{Baseline characteristics}

One hundred and fifty-four patients were randomized to 4 groups ( 38 to placebo, 41 to carvedilol $6.25 \mathrm{mg}$ OD, 38 to carvedilol $12.5 \mathrm{mg}$ OD and 37 to carvedilol $25 \mathrm{mg}$ OD); their baseline characteristics are summarized in Table 1. Patient characteristics, cardiac function, cancer type and cumulative doxorubicin dose was similar amongst treatment groups.

\subsection{Reproducibility assessment}

Based on baseline data, the intra-observer variability, ICC, was high: 0.975 (95\% CI: 0.926-0.991) and the inter-observer variability was 0.809 (95\% CI: 0.509-0.925) for LVEF. Similarly, for follow-up data, the intra and inter ICC were 0.955 (95\% CI: 0.887-0.982) and 0.982 (95\% CI: 0.995-0.993), respectively.

\subsection{Treatment outcomes at end of six months}

At 6 months, four (11\%) patients assigned to placebo but only one (1\%) assigned to carvedilol $12.5 \mathrm{mg}$ had an $\mathrm{LVEF}<50 \%$ $(\mathrm{p}=0.013)$.

The cumulative doxorubicin dose in the five patients who developed an LVEF < 50\% were: $160 \mathrm{mg} / \mathrm{m}^{2}$ (one patient), $300 \mathrm{mg} /$ $\mathrm{m}^{2}$ (two patients) and $360 \mathrm{mg} / \mathrm{m}^{2}$ (one patient) in the placebo group and $240 \mathrm{mg} / \mathrm{m}^{2}$ in one patient who received carvedilol $12.5 \mathrm{mg}$.

By six months, for patients assigned to placebo, LVEF had fallen by $3.8 \pm 7.1 \%(p=0.002)$ with $45 \%$ having some reduction in LVEF. In contrast, there was no significant decline in LVEF for any group assigned to carvedilol [6.25 mg ( $p=0.059), 12.5 \mathrm{mg}$ $(\mathrm{p}=0.100)$, or $25 \mathrm{mg}(\mathrm{p}=0.073)]$ and depicted as bar and line graphs in Figs. 1 and 2. On inter-group comparison, no significant change was observed in LVEF between placebo and all carvedilol groups $(\mathrm{p}=0.185)$. Similarly, no difference noted in the inter group comparison with any carvedilol dose: $6.25 \mathrm{mg}(\mathrm{p}=0.080) ; 12.5 \mathrm{mg}$ $(\mathrm{p}=0.219)$; or $25 \mathrm{mg}(\mathrm{p}=0.060)$.

\subsection{Effect of doxorubicin on $L V$ size}

At 6-months there was an increase in mean LVSd in patients assigned to placebo from baseline of $28 \pm 4 \mathrm{~mm}$ to $31 \pm 6 \mathrm{~mm}$ $(\mathrm{p}=0.002)$ but not amongst patients assigned to carvedilol. The differences between placebo and carvedilol, either in individual dose groups or overall were not significant [6.25 mg ( $29 \pm 4$ vs. $30 \pm 3, \mathrm{p}=0.059) ; 12.5 \mathrm{mg}(29 \pm 4$ vs. $30 \pm 4, \mathrm{p}=0.178) ; 25 \mathrm{mg}$ $(30 \pm 5$ vs. $31 \pm 4, p=0.459)]$. The mean changes in LVDd for patients assigned to placebo or carvedilol were similar (Table 2).

\subsubsection{Effect of doxorubicin on diastolic function}

E' decreased only in patients treated with carvedilol $12.5 \mathrm{mg}$ or carvedilol $25 \mathrm{mg}$ (Table 2). However, mean E' remained above 10 and mean $E / E^{\prime}$ ratio below 13 in all groups $(8.0 \pm 4.0)$. Furthermore, 
Table 1

Baseline Clinical Characteristics of Patients.

\begin{tabular}{|c|c|c|c|c|c|}
\hline & \multicolumn{4}{|l|}{ Carvedilol } & \multirow[t]{2}{*}{ p-value } \\
\hline & Placebo $(n=38)$ & $6.25 \mathrm{mg}(\mathrm{n}=41)$ & $12.5 \mathrm{mg}(\mathrm{n}=38)$ & $25 \mathrm{mg}(\mathrm{n}=37)$ & \\
\hline Age (yrs) & $40.4 \pm 14.0$ & $46.1 \pm 13.0$ & $41.3 \pm 18.2$ & $42.0 \pm 15.0$ & 0.345 \\
\hline Women (\%) & 76 & 80 & 58 & 77 & 0.119 \\
\hline BMI $\left(\mathrm{kg} / \mathrm{m}^{2}\right)$ & $27.4 \pm 6.5$ & $29.1 \pm 6.3$ & $27.1 \pm 6.4$ & $28.3 \pm 7.2$ & 0.511 \\
\hline Baseline EF (\%) & $62.0 \pm 4.6$ & $61.4 \pm 3.9$ & $60.0 \pm 4.2$ & $60.5 \pm 4.2$ & 0.177 \\
\hline LVDd (mm) & $45.3 \pm 5.3$ & $46.0 \pm 5.1$ & $44.8 \pm 4.3$ & $44.6 \pm 6.3$ & 0.634 \\
\hline LVSd (mm) & $28.0 \pm 4.4$ & $29.3 \pm 4.4$ & $29.2 \pm 4.2$ & $30.2 \pm 5.7$ & 0.243 \\
\hline $\mathrm{E} / \mathrm{A}(\%)$ & $1.2 \pm 0.4$ & $1.2 \pm 0.5$ & $1.3 \pm 0.5$ & $1.2 \pm 0.3$ & 0.836 \\
\hline $\mathrm{E}^{\prime}$ & $11.3 \pm 3.0$ & $11.9 \pm 3.2$ & $13.2 \pm 5.1$ & $11.8 \pm 3.4$ & 0.150 \\
\hline DT & $190.8 \pm 33.8$ & $200.6 \pm 28.2$ & $203.6 \pm 38.7$ & $199.5 \pm 38.8$ & 0.421 \\
\hline $\mathrm{E} / \mathrm{E}^{\prime}$ & $7.2 \pm 2.6$ & $7.4 \pm 3.0$ & $7.0 \pm 3.3$ & $6.9 \pm 2.6$ & 0.836 \\
\hline \multicolumn{6}{|l|}{ Cancer Type (\%) } \\
\hline Breast & $20(53)$ & $23(56)$ & $13(34)$ & $16(43)$ & 0.208 \\
\hline NHL & $5(13)$ & $8(20)$ & $11(29)$ & $12(33)$ & 0.177 \\
\hline Other & $13(34)$ & $10(24)$ & $14(37)$ & $9(24)$ & 0.502 \\
\hline Cum Doxo dose $\left(\mathrm{mg} / \mathrm{m}^{2}\right)$ & $265.6 \pm 98.5$ & $252 \pm 65.0$ & $282 \pm 78.5$ & $261.0 \pm 101.8$ & 0.473 \\
\hline Hypertension & 4 & 4 & 3 & 7 & 0.524 \\
\hline Diabetes Mellitus & 6 & 8 & 5 & 8 & 0.712 \\
\hline Dyslipidemia & 2 & 0 & 3 & 3 & 0.267 \\
\hline
\end{tabular}

Data are mean \pm standard deviation or percentage. Cum Doxo dose and NHL denote cumulative doxorubicin dose and Non-Hodgkin Lymphoma respectively.

$P$-value by ANOVA for continuous variables and Chi Square or Fisher's test for categorical variables.

no significant differences in Doppler echocardiography variables were observed for patients assigned to placebo or carvedilol.

\subsection{Mortality}

Overall 11 patients died. Two deaths (5.3\%) occurred on placebo, one $(2.4 \%)$ on carvedilol $6.25 \mathrm{mg}$, four $(10.5 \%)$ on carvedilol $12.5 \mathrm{mg}$, and four (10.8\%) on carvedilol $25 \mathrm{mg}$. Of these, 8 died in hospital which was attributed to end stage cancer with metastasis in addition to respiratory failure and massive pleural effusion in some cases; and sepsis in other patients. We did not find any significant difference in the hemodynamic response (blood pressure and heart rate) between all carvedilol doses (see Table 4).

\section{Discussion}

This study provides further evidence that carvedilol may provide protection against anthracycline-induced cardiomyopathy. Moreover, this effect may be observed with a broad range of carvedilol doses. This latter observation is important because the side effects of beta-blockers appear strongly dose-related ${ }^{12}$ and cancer patients may experience symptoms that might be ascribed

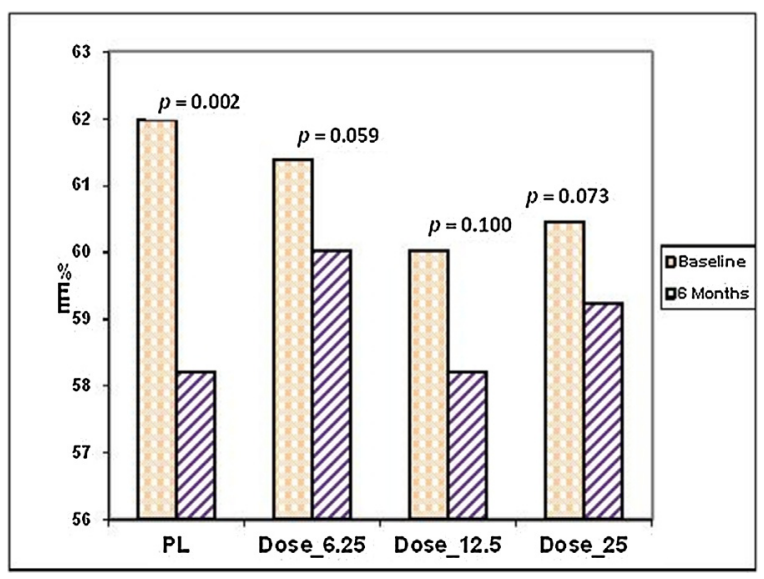

Fig. 1. LVEF at baseline and 6 months among different doses. to higher doses of beta-blocker. Lower doses of carvedilol may offer myocardial protection but with fewer side effects.

Earlier, smaller randomized studies by Kalay et al. and Salehi et al. suggested that carvedilol offered myocardial protection to cancer patients treated with anthracyclines. ${ }^{9,10}$ Kalay et al. investigated the protective effect of prescribing carvedilol $12.5 \mathrm{mg}$ once daily to 50 patients treated with doxorubicin or epirubicin. ${ }^{9}$ There was no attempt at blinding. Measures of both systolic and diastolic function deteriorated less amongst patients assigned to carvedilol. Salehi et al. conducted a study in 66 patients treated with doxorubicin or epirubicin who were randomized to receive placebo, carvedilol $12.5 \mathrm{mg}$ once daily or carvedilol $25 \mathrm{mg}$ once daily. The paper does not explicitly state whether or not the study was blinded. Overall, there was no significant benefit with carvedilol but the authors claimed that the lower dose was associated with improved diastolic function and the higher dose with both improved diastolic and systolic function. The prevention of Cardiac Dysfunction During Adjuvant Breast Cancer Therapy (PRADA) trial results were recently reported. They studied women with breast cancer in a double-blind, placebocontrolled study with approximately 30 patients per group assigned either to placebo, metoprolol succinate titrated to $100 \mathrm{mg} /$ day or candesartan titrated to $32 \mathrm{mg} / \mathrm{day}$. Compared to those assigned to placebo, patients assigned to candesartan had a smaller decline in LVEF after chemotherapy $(p=0.03)$ but did not observe a similar effect with metoprolol and questioned if other beta-blockers, such as carvedilol, might have led to different results. ${ }^{13}$

Our study differs from previous trials of carvedilol in several important ways. This is the first unequivocally, double-blind, randomized, placebo-controlled study to investigate the effects of carvedilol in patients treated with anthracyclines. It is the only study to evaluate three doses of carvedilol and is also substantially larger than the previous two studies (Table 3$)^{9,10}$ In previous randomized trials, the mean cumulative doxorubicin dose was $\geq 500 \mathrm{mg} / \mathrm{m}^{2}$ but only four patients received a cumulative doxorubicin dose of $>400 \mathrm{mg} / \mathrm{m}^{2}$ in our study, which may explain the lower incidence of LV dysfunction in the control group in our study. Doxorubicin cardiotoxicity is cumulative and typically occurs at an average total dose of $>500 \mathrm{mg} / \mathrm{m}^{2}$ but can occur at cumulative doses as low as $300 \mathrm{mg} / \mathrm{m}^{2}$.

During six months of chemotherapy, four (11\%) patients in the placebo group but only one on carvedilol (3\%) developed LV systolic dysfunction (LVSD). Kalay et al. observed the development of LVSD 

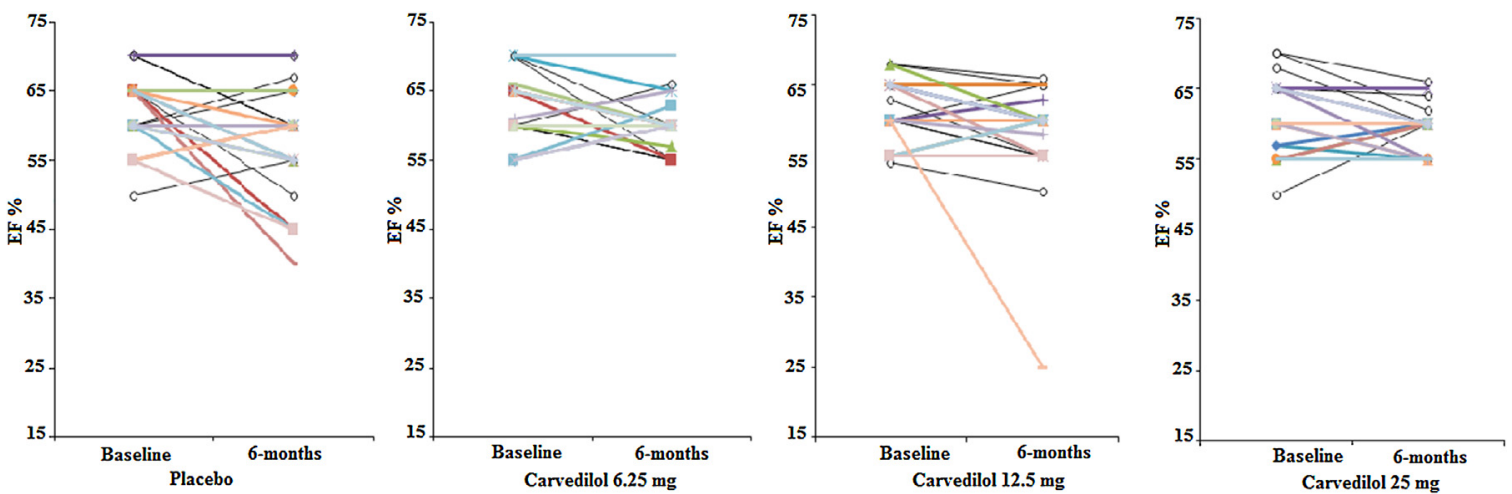

Fig. 2. Individual left ventricle ejection fraction at baseline and 6-months.

Table 2

Comparison of echocardiography and tissue Doppler variables at baseline and 6 month of follow-up.

\begin{tabular}{|c|c|c|c|c|c|c|c|c|c|}
\hline & & Placebo & $p$ value & Car (6.25 mg) & $p$ value & Car (12.5 mg) & $p$ value & Car (25 mg) & $p$ value \\
\hline \multirow[t]{2}{*}{ LVSd } & Baseline & $28.0 \pm 4.4$ & 0.002 & $29.3 \pm 4.4$ & 0.059 & $29.2 \pm 4.2$ & 0.178 & $30.2 \pm 5.7$ & 0.458 \\
\hline & 6 months & $30.7 \pm 5.7$ & & $30.4 \pm 3.0$ & & $30.3 \pm 4.3$ & & $30.9 \pm 4.1$ & \\
\hline \multirow[t]{2}{*}{ LVDd } & Baseline & $45.3 \pm 5.3$ & 0.566 & $46.0 \pm 5.1$ & 0.166 & $44.8 \pm 4.3$ & 0.011 & $44.6 \pm 6.3$ & 0.368 \\
\hline & 6 months & $45.9 \pm 7.5$ & & $46.8 \pm 4.0$ & & $46.0 \pm 3.7$ & & $45.5 \pm 5.3$ & \\
\hline \multirow[t]{2}{*}{$\mathrm{EF}$} & Baseline & $62.0 \pm 4.6$ & 0.002 & $61.4 \pm 3.9$ & 0.059 & $60.0 \pm 4.1$ & 0.100 & $60.4 \pm 4.2$ & 0.073 \\
\hline & 6 months & $58.2 \pm 6.6$ & & $60.0 \pm 2.9$ & & $58.2 \pm 6.6$ & & $59.2 \pm 2.8$ & \\
\hline \multirow[t]{2}{*}{$E^{\prime}$} & Baseline & $11.3 \pm 3.0$ & 0.365 & $11.9 \pm 3.2$ & 0.067 & $13.2 \pm 5.0$ & 0.001 & $11.8 \pm 3.4$ & 015 \\
\hline & 6 months & $10.9 \pm 3.8$ & & $10.9 \pm 3.4$ & & $11.8 \pm 4.9$ & & $10.8 \pm 3.0$ & \\
\hline \multirow[t]{2}{*}{$\mathrm{E} / \mathrm{A}$} & Baseline & $1.22 \pm 0.4$ & 0.533 & $1.20 \pm 0.5$ & 0.359 & $1.26 \pm 0.5$ & 0.949 & $1.17 \pm 0.3$ & 0.251 \\
\hline & 6 months & $1.20 \pm 0.4$ & & $1.14 \pm 0.4$ & & $1.27 \pm 0.6$ & & $1.13 \pm 0.3$ & \\
\hline \multirow[t]{2}{*}{ DT } & Baseline & $190.7 \pm 33.9$ & 0.188 & $200.8 \pm 28.2$ & 0.637 & $203.6 \pm 38.7$ & 0.228 & $199.5 \pm 38.8$ & 0.139 \\
\hline & 6 months & $199.7 \pm 39.8$ & & $197.1 \pm 30.0$ & & $213.3 \pm 45.1$ & & $209.3 \pm 40.9$ & \\
\hline \multirow[t]{2}{*}{$\mathrm{E} / \mathrm{E}^{\prime}$} & Baseline & $7.2 \pm 2.6$ & 0.413 & $7.4 \pm 3.0$ & 0.730 & $7.0 \pm 3.3$ & 0.033 & $6.9 \pm 2.6$ & 0.788 \\
\hline & 6 months & $7.6 \pm 3.3$ & & $7.6 \pm 3.6$ & & $8.0 \pm 4.0$ & & $7.0 \pm 2.2$ & \\
\hline
\end{tabular}

Car* stands for carvedilol.

Table 3

Comparison of studies on the prophylactic use of carvedilol in adult patients receiving anthracycline

\begin{tabular}{|c|c|c|c|c|}
\hline & \multicolumn{4}{|l|}{ Studies } \\
\hline & Present & Kalay et al. & Salehi et al. & Total \\
\hline \multicolumn{5}{|l|}{ Study Design } \\
\hline Single center & Yes & Yes & Yes & \\
\hline Placebo controlled & Yes & Yes & Yes & \\
\hline Blind & Double & Single & NA & \\
\hline Treatment Groups & 4 & 2 & 3 & \\
\hline \multicolumn{5}{|l|}{ Sample Size } \\
\hline Placebo & 38 & 25 & 22 & 85 \\
\hline Carvedilol $6.25 \mathrm{mg}$ od & 41 & - & - & 41 \\
\hline Carvedilol $12.50 \mathrm{mg}$ od & 38 & 25 & 22 & 75 \\
\hline Carvedilol $25.00 \mathrm{mg}$ od & 37 & - & 22 & 59 \\
\hline Chemotherapy Treatment & Doxorubicin & Doxorubicin or epirubicin & Doxorubicin or epirubicin & \\
\hline Cumulative Doxorubicin dose & $<400 \mathrm{mg} / \mathrm{m}^{2}$ & $\sim 520 \mathrm{mg} / \mathrm{m}^{2}$ & $\sim 530 \mathrm{mg} / \mathrm{m}^{2}$ & \\
\hline Follow up period & 6 months & 6 months & 4 months & \\
\hline Echo studies & $\begin{array}{l}\text { Baseline, then at } 2,4 \& 6 \\
\text { months. }\end{array}$ & $\begin{array}{l}\text { Baseline then after } \\
\text { chemotherapy. }\end{array}$ & $\begin{array}{l}\text { Baseline, then only at } 4 \\
\text { months. }\end{array}$ & \\
\hline Beneficial effect of carvedilol on LV function & Yes & Yes & Yes & \\
\hline Number of patients who developed & Placebo: $4(11 \%)$ & Placebo: 5 (20\%) & Placebo: 5 (23\%). & $14 / 85(16 \%)$ \\
\hline cardiomyopathy & Carvedilol: $1(1 \%)(p=0.013)$. & Carvedilol: $1(4 \%)$ & Carvedilol: 6 (14\%) & $8 / 145(6 \%)$ \\
\hline
\end{tabular}

in one patient (4\%) treated with carvedilol and 5 patients (23\%) assigned to placebo and Salehi et al. in five patients (22.7\%) treated with carvedilol $12.5 \mathrm{mg} /$ day but in only one (5\%) treated with carvedilol $25 \mathrm{mg} /$ day and $5(23 \%)$ patients in control group. Cumulatively, including the current study, 14 of 85 patients (16\%) assigned to placebo developed LVSD compared to 8 of 145 patients (6\%) assigned to carvedilol ( $\mathrm{p}<0.01)$.
We found no evidence to support a beneficial effect of carvedilol on diastolic dysfunction; the incidence of different grades of diastolic dysfunction were similar across treatment groups. This finding differs from those reported in earlier studies. ${ }^{9,10}$ At 6 months, there was an increase in the $E / E^{\prime}$, which was statistically significant in the carvedilol $12.5 \mathrm{mg}$ group when compared to baseline. In the PRADA study, the increase in E/E' on metoprolol 
Table 4

Blood pressure and heart rate at baseline and 6 months for the 4 groups.

\begin{tabular}{|c|c|c|c|c|c|c|c|}
\hline \multirow[t]{2}{*}{ Group Dose } & & \multicolumn{2}{|c|}{ Heart Rate } & \multicolumn{2}{|c|}{ Systolic BP } & \multicolumn{2}{|c|}{ Diastolic BP } \\
\hline & & Baseline & 6-month & Baseline & 6-month & Baseline & 6-month \\
\hline Placebo & Mean & 89.33 & 86.88 & 120.49 & 112.76 & 72.84 & 73.92 \\
\hline Carvedilol $6.25 \mathrm{mg}$ & Mean & 87.08 & 84.89 & 129.47 & 119.64 & 74.44 & 73.21 \\
\hline Carvedilol $12.5 \mathrm{mg}$ & Mean & 85.26 & 77.56 & 121.71 & 112.59 & 72.97 & 70.12 \\
\hline Carvedilol $25 \mathrm{mg}$ & Mean & 85.45 & 81.79 & 120.53 & 116.78 & 72.41 & 74.11 \\
\hline
\end{tabular}

succinate was highly significant. Sympathetic activation increases the velocity of myocardial relaxation and so it is not surprising that beta-blockers may slow the rate of ventricular relaxation.

In clinical trials of heart failure, carvedilol was usually given twice daily and in total daily doses of up to $100 \mathrm{mg} /$ day. Whether other doses or dosing regimens would carry greater benefit than we observed should be considered in other trials. Whether, heart rate rather than dose achieved is the more important target for beta-blocker therapy is also uncertain. ${ }^{12-14}$ It is also unclear whether similar effects would be observed with other betablockers. Neither the study size nor the mechanistic character of the primary outcome (change in LVEF) is sufficient to provide conclusive evidence of clinically relevant benefits with carvedilol for this population. However, this study does give hope for patients with few alternatives, adds to the existing evidence-base and provides valuable data to inform the design of definitive multicentre trials.

\subsection{Study limitations}

While this is the largest trial on the topic, including men and women with different doses of carvedilol, it was still underpowered to be able to detect a difference in outcomes between treatment groups. Data was missed for troponin and creatinine from a good percentage of patients. Long-term outcome was not assessed.

\subsection{Conclusion}

These data provide further evidence suggesting that prophylactic use of carvedilol might attenuate or prevent the decline in LVEF associated with the use of anthracyclines for the treatment of cancer.

\section{Conflicts of interest}

None.

\section{Acknowledgements}

Carvedilol $^{\circledR}$.
Saudi Pharmaceutical Industries \& Medical Appliances Corporation (SPIMACO ADDWAEIH). They supplied us with the different carvedilol doses and the placebo.

\section{Appendix A. Supplementary data}

Supplementary material related to this article can be found, in the online version, at doi:https://doi.org/10.1016/j.ihj.2018.06.011.

\section{References}

1. Cardinale Daniela, Colombo Alessandro, Bacchiani Giulia, et al. Early detection of anthracycline cardiotoxicity and improvement with heart failure therapy. Circulation. 2015;131(22):1981-1988.

2. Pfeffer B, Tziros C, Katz Br R. J Cardiol. 2009;16:85-89.

3. Pfeffer B, Tziros C, Katz R. Current concepts of anthracycline cardiotoxicity: pathogenesis, diagnosis and prevention. Br J Cardiol. 2009;16:85-89.

4. Hequet $\mathrm{O}$, Le $\mathrm{OH}$, Moullet I, et al. Subclinical late cardiomyopathy after doxorubicin therapy for lymphoma in adults. J Clin Oncol. 2004;22:1864-1871.

5. Sawaya H, Sebag IA, Plana JC, et al. Early detection and prediction of cardiotoxicity in chemotherapy-treated patients. Am J Cardiol. 2011;107:13751380.

6. Carreira RS, Monteiro P, Gon Alves LM, Providencia LA. Carvedilol: just another beta-blocker or a powerful cardioprotector? Cardiovasc Hematol Disord Drug Targets. 2006:6:57-66.

7. Jaylo MSN, Narraval C, Chiew B, Lapuz F. The cardioprotective effects of angiotensin converting enzyme inhibitor (perindopril) as prophylaxis of doxorubicin-induced cardiomyopathy-a pilot study. Intern Med Phil J. 2006;44:75-82.

8. Hellmann K. Preventing the cardiotoxicity of anthracyclines by dexrazoxane BMJ. 1999;319:1085-1086.

9. Kalay N, Basar E, Ozdogru I, et al. Protective effects of carvedilol against anthracycline-induced cardiomyopathy. J Am Coll Cardiol. 2006;48:22582262.

10. Salehi R, Zamani B, Esfehani A, Ghafari S, Abasnezhad M, Goldust M. Protective effect of carvedilol in cardiomyopathy caused by anthracyclines in patients suffering from breast cancer and lymphoma. Am Heart Hosp J. 2011;9:95-98.

11. Nagueh SF, Appleton CP, Gillebert TC, et al. Recommendations for the evaluation of left ventricular diastolic function by echocardiography. JASE. 2009;22:107-133.

12. Cleland JG, Coletta AP, Freemantle N, Ahmed D, Rubis P, Clark AL. Clinical trials update from the American Heart Association Meeting 2009: HEAAL, FAIR-HF, J-CHF, HeartMate II, PACE and a meta-analysis of dose-ranging studies of betablockers in Heart failure. Eur J Heart Fail. 2010;12:197-201.

13. Gulati G, Heck SL, Ree AH, et al. Prevention of cardiac dysfunction during adjuvant breast cancer therapy (PRADA): a $2 \times 2$ factorial, randomized, placebo-controlled, double-blind clinical trial of candesartan and metoprolol Eur Heart J. 2016;37:1671-1680.

14. Cullington D, Goode KM, Clark AL, Cleland JG. Heart rate or beta-bloker dose in patients with chronic heart failure: which is the better target? Eur J Heart Fail. 2012;14:737-747. 